International Journal of Computer Science, Engineering and Applications (IJCSEA) Vol.2, No.1, February 2012

\title{
The Design OF AdAPTIVE CONTROLLER AND SYNCHRONIZER FOR QI-CHEN SYSTEM WITH UNKNOWN PARAMETERS
}

\author{
Sundarapandian Vaidyanathan ${ }^{1}$ \\ ${ }^{1}$ Research and Development Centre, Vel Tech Dr. RR \& Dr. SR Technical University \\ Avadi, Chennai-600 062, Tamil Nadu, INDIA \\ sundarvtuegmail.com
}

\begin{abstract}
This paper investigates the design problem of adaptive controller and synchronizer for the Qi-Chen system (2005), when the system parameters are unknown. First, we build an adaptive controller to stabilize the QiChen chaotic system to its unstable equilibrium at the origin. Then we build an adaptive synchronizer to achieve global chaos synchronization of the identical Qi-Chen chaotic systems with unknown parameters. The results derived for adaptive stabilization and adaptive synchronization for the Qi-Chen chaotic system are established using adaptive control theory and Lyapunov stability theory. Numerical simulations have been shown to demonstrate the effectiveness of the adaptive control and synchronization schemes derived in this paper for the Qi-Chen chaotic system.
\end{abstract}

\section{KEYWORDS}

Adaptive Control, Chaos, Chaotic Systems, Synchronization, Qi-Chen System.

\section{INTRODUCTION}

Chaotic systems are nonlinear dynamical systems with the following characteristics: extreme sensitivity to changes in initial conditions, random-like behaviour, deterministic motion, trajectories of chaotic systems pass through any point an infinite number of times. Experimentally, chaos was first discovered by Lorenz ([1], 1963) while he was simulating weather models. A chaotic system simpler than the Lorenz system was proposed by Rössler ([2], 1976). The theoretical equations of the Rössler system were later found to be useful in modelling equilibrium in chemical reactions.

The control of chaotic systems is to design state feedback control laws that stabilizes the chaotic systems around the unstable equilibrium points. Active control technique is used when the system parameters are known and adaptive control technique is used when the system parameters are unknown [3-4].

Synchronization of chaotic systems is a phenomenon that may occur when two or more chaotic attractors are coupled or when a hyperchaotic attractor drives another hyperchaotic attractor. In the last two decades, there has been significant interest in the literature on the synchronization of chaotic and hyperchaotic systems [5-16]. 
In 1990, Pecora and Carroll [5] introduced a method to synchronize two identical chaotic systems and showed that it was possible for some chaotic systems to be completely synchronized. From then on, chaos synchronization has been widely explored in a variety of fields including physical systems [6], chemical systems [7], ecological systems [8], secure communications [9-10], etc.

In most of the chaos synchronization approaches, the master-slave or drive-response formalism has been used. If a particular chaotic system is called the master or drive system and another chaotic system is called the slave or response system, then the idea of synchronization is to use the output of the master system to control the slave system so that the output of the slave system tracks the output of the master system asymptotically.

The seminal work by Pecora and Carroll (1990) has been followed by a variety of impressive approaches in the literature such as the sampled-data feedback method [11], OGY method [12], time-delay feedback method [13], backstepping method [14], active control method [15-20], adaptive control method [21-25], sliding mode control method [26-28], etc.

This paper is organized as follows. In Section 2, we give a description of the Qi-Chen chaotic system (Qi, Chen, Du, Chen and Yuan, [29], 2005). In Section 3, we derive results for the adaptive control of Qi-Chen chaotic system with unknown parameters. In Section 4, we derive results for the adaptive synchronization of the identical Qi-Chen chaotic systems with unknown parameters. Section 5 contains a summary of the main results derived in this paper.

\section{SYSTEM DESCRIPTION}

The Qi-Chen system ([29], 2005) is described by the 3D dynamics

$$
\begin{aligned}
& \dot{x}_{1}=a\left(x_{2}-x_{1}\right)+x_{2} x_{3} \\
& \dot{x}_{2}=c x_{1}-x_{2}-x_{1} x_{3} \\
& \dot{x}_{3}=x_{1} x_{2}-b x_{3}
\end{aligned}
$$

where $x_{1}, x_{2}, x_{3}$ are the state variables of the system and $a, b, c$ are constant, positive parameters of the system.

The system (1) is chaotic when the parameter values are taken as

$$
a=35, b=8 / 3 \text { and } c=80
$$

Figure 1 describes the strange attractor of the Qi-Chen chaotic system (1).

When the parameter values are taken as in (2) for the Qi-Chen chaotic system (1), the system linearization matrix at the equilibrium point $E_{0}=(0,0,0)$ is given by

$$
A=\left[\begin{array}{ccc}
-35 & 35 & 0 \\
80 & -1 & 0 \\
0 & 0 & -8 / 3
\end{array}\right]
$$

which has the eigenvalues

$$
\lambda_{1}=-73.5788, \quad \lambda_{2}=-2.6667, \quad \lambda_{3}=37.5788
$$


Since $\lambda_{3}$ is a positive eigenvalues of $A$, it follows from Lyapunov stability theory [30] that the Qi-Chen system (1) is unstable at the equilibrium point $E_{0}=(0,0,0)$.

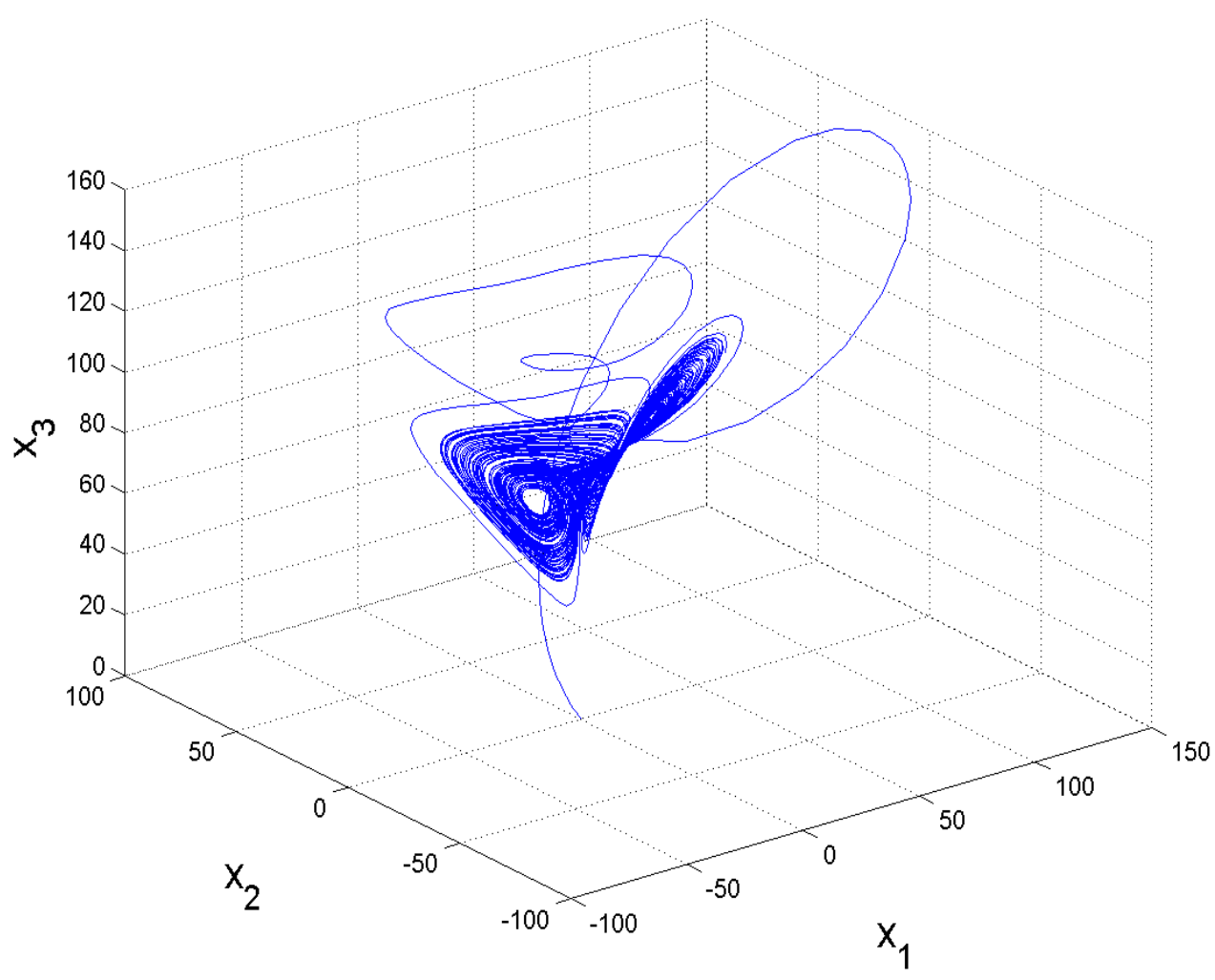

Figure 1. The Strange Attractor of the Qi-Chen Chaotic System

\section{AdAPTIVE ConTrol of THE QI-Chen ChaOtic System}

\subsection{Theoretical Results}

In this section, we design adaptive control law for globally stabilizing the Qi-Chen system (2005), when the parameter values are unknown.

Thus, we consider the controlled Qi-Chen system, which is described by the 3D dynamics

$$
\begin{aligned}
& \dot{x}_{1}=a\left(x_{2}-x_{1}\right)+x_{2} x_{3}+u_{1} \\
& \dot{x}_{2}=c x_{1}-x_{2}-x_{1} x_{3}+u_{2} \\
& \dot{x}_{3}=x_{1} x_{2}-b x_{3}+u_{3}
\end{aligned}
$$


where $u_{1}, u_{2}$ and $u_{3}$ are feedback controllers to be designed using the states $x_{1}, x_{2}, x_{3}$ and estimates $\hat{a}, \hat{b}, \hat{c}$ of the unknown parameters $a, b, c$ of the system.

In order to ensure that the controlled system (3) globally converges to the origin asymptotically, we consider the following adaptive control functions

$$
\begin{aligned}
& u_{1}=-\hat{a}\left(x_{2}-x_{1}\right)-x_{2} x_{3}-k_{1} x_{1} \\
& u_{2}=-\hat{c} x_{1}+x_{2}+x_{1} x_{3}-k_{2} x_{2} \\
& u_{3}=-x_{1} x_{2}+\hat{b} x_{3}-k_{3} x_{3}
\end{aligned}
$$

where $\hat{a}, \hat{b}$ and $\hat{c}$ are estimates of the parameters $a, b$ and $c$, respectively, and $k_{i},(i=1,2,3)$ are positive constants.

Substituting the control law (4) into the controlled Qi-Chen dynamics (3), we obtain

$$
\begin{aligned}
& \dot{x}_{1}=(a-\hat{a})\left(x_{2}-x_{1}\right)-k_{1} x_{1} \\
& \dot{x}_{2}=(c-\hat{c}) x_{1}-k_{2} x_{2} \\
& \dot{x}_{3}=-(b-\hat{b}) x_{3}-k_{3} x_{3}
\end{aligned}
$$

Let us now define the parameter errors as

$$
e_{a}=a-\hat{a}, \quad e_{b}=b-\hat{b} \text { and } e_{c}=c-\hat{c}
$$

Using (6), the closed-loop dynamics (5) can be written compactly as

$$
\begin{aligned}
& \dot{x}_{1}=e_{a}\left(x_{2}-x_{1}\right)-k_{1} x_{1} \\
& \dot{x}_{2}=e_{c} x_{1}-k_{2} x_{2} \\
& \dot{x}_{3}=-e_{b} x_{3}-k_{3} x_{3}
\end{aligned}
$$

For the derivation of the update law for adjusting the parameter estimates $\hat{a}, \hat{b}$ and $\hat{c}$, the Lyapunov approach is used.

Consider the quadratic Lyapunov function

$$
V\left(x_{1}, x_{2}, x_{3}, e_{a}, e_{b}, e_{c}\right)=\frac{1}{2}\left(x_{1}^{2}+x_{2}^{2}+x_{3}^{2}+e_{a}^{2}+e_{b}^{2}+e_{c}^{2}\right)
$$

which is a positive definite function on $R^{6}$.

Note also that

$$
\dot{e}_{a}=-\dot{\hat{a}}, \quad \dot{e}_{b}=-\dot{\hat{b}}, \dot{e}_{c}=-\dot{\hat{c}}
$$

Differentiating $V$ along the trajectories of (7) and using (9), we obtain 
International Journal of Computer Science, Engineering and Applications (IJCSEA) Vol.2, No.1, February 2012

$$
\dot{V}=-k_{1} x_{1}^{2}-k_{2} x_{2}^{2}-k_{3} x_{3}^{2}+e_{a}\left[x_{1}\left(x_{2}-x_{1}\right)-\dot{\hat{a}}\right]+e_{b}\left(-x_{3}^{2}-\dot{\hat{b}}\right)+e_{c}\left(x_{1} x_{2}-\dot{\hat{c}}\right)
$$

In view of Eq. (10), the estimated parameters are updated by the following law:

$$
\begin{aligned}
& \dot{\hat{a}}=x_{1}\left(x_{2}-x_{1}\right)+k_{4} e_{a} \\
& \dot{\hat{b}}=-x_{3}^{2}+k_{5} e_{b} \\
& \dot{\hat{c}}=x_{1} x_{2}+k_{6} e_{c}
\end{aligned}
$$

where $k_{4}, k_{5}$ and $k_{6}$ are positive constants.

Substituting (11) into (10), we get

$$
\dot{V}=-k_{1} x_{1}^{2}-k_{2} x_{2}^{2}-k_{3} x_{3}^{2}-k_{4} e_{a}^{2}-k_{5} e_{b}^{2}-k_{6} e_{c}^{2}
$$

which is a negative definite function on $R^{6}$.

Thus, by Lyapunov stability theory [30], we obtain the following result.

Theorem 1. The controlled Qi-Chen system (1) with unknown parameters is globally and exponentially stabilized for all initial conditions $x(0) \in R^{3}$ by the adaptive control law (4), where the update law for the parameters is given by $(11)$ and $k_{i},(i=1, \ldots, 6)$ are positive constants.

\subsection{Numerical Results}

For the numerical simulations, the fourth order Runge-Kutta method is used to solve the chaotic system (3) with the adaptive control law (4) and the parameter update law (11).

The parameters of the Qi-Chen system (3) are selected as $a=35, b=8 / 3$ and $c=80$.

For the adaptive and update laws, we take $k_{i}=5, \quad(i=1,2, \ldots, 6)$.

Suppose that the initial values of the estimated parameters are

$$
\hat{a}(0)=6, \hat{b}(0)=18, \hat{c}(0)=5
$$

The initial state of the controlled Qi-Chen system (3) is taken as

$$
x_{1}(0)=21, \quad x_{2}(0)=-16, \quad x_{3}(0)=30
$$

When the adaptive control law (4) and the parameter update law (11) are used, the controlled modified hyperchaotic Lü system converges to the equilibrium $E_{0}=(0,0,0)$ exponentially as shown in Figure 2. 
International Journal of Computer Science, Engineering and Applications (IJCSEA) Vol.2, No.1, February 2012

The time-history of the parameter estimates is shown in Figure 3. The time-history of the parameter estimation errors is shown in Figure 4.

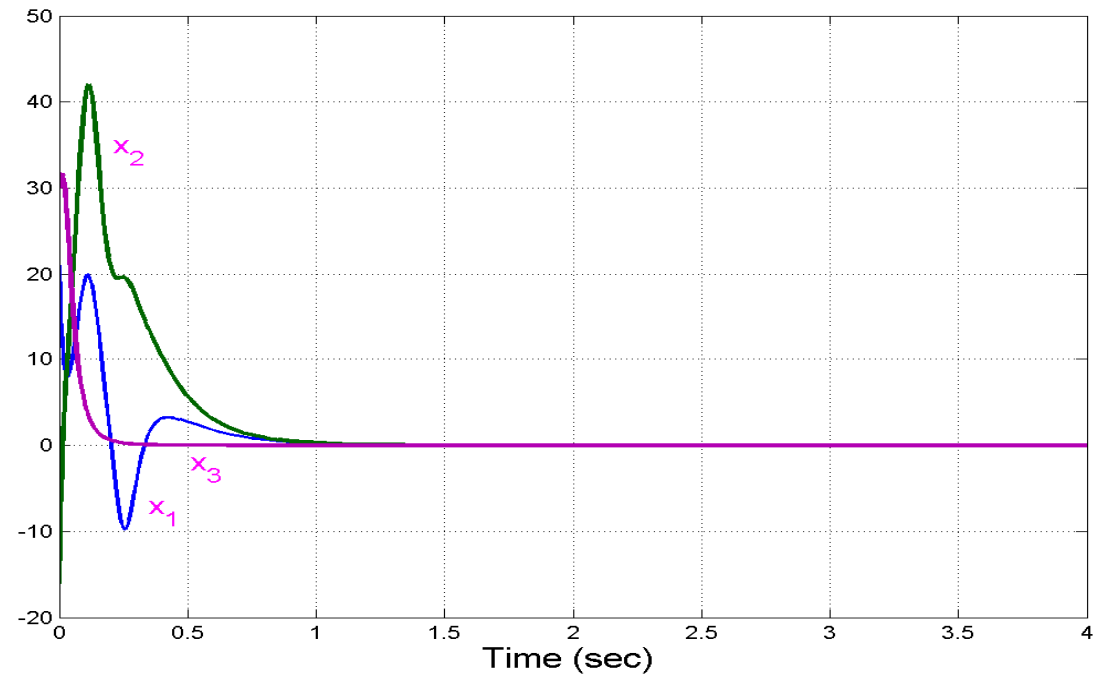

Figure 2. Time Responses of the Controlled Qi-Chen System

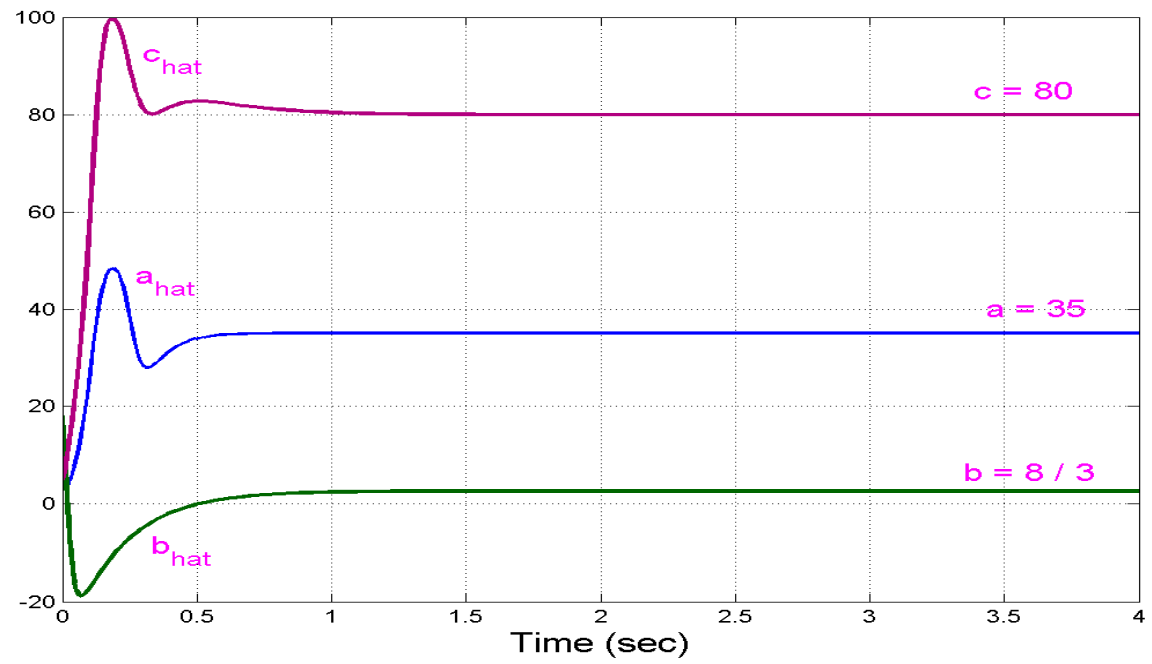

Figure 3. Time-History of the Parameter Estimates $\hat{a}(t), \hat{b}(t), \hat{c}(t)$ 


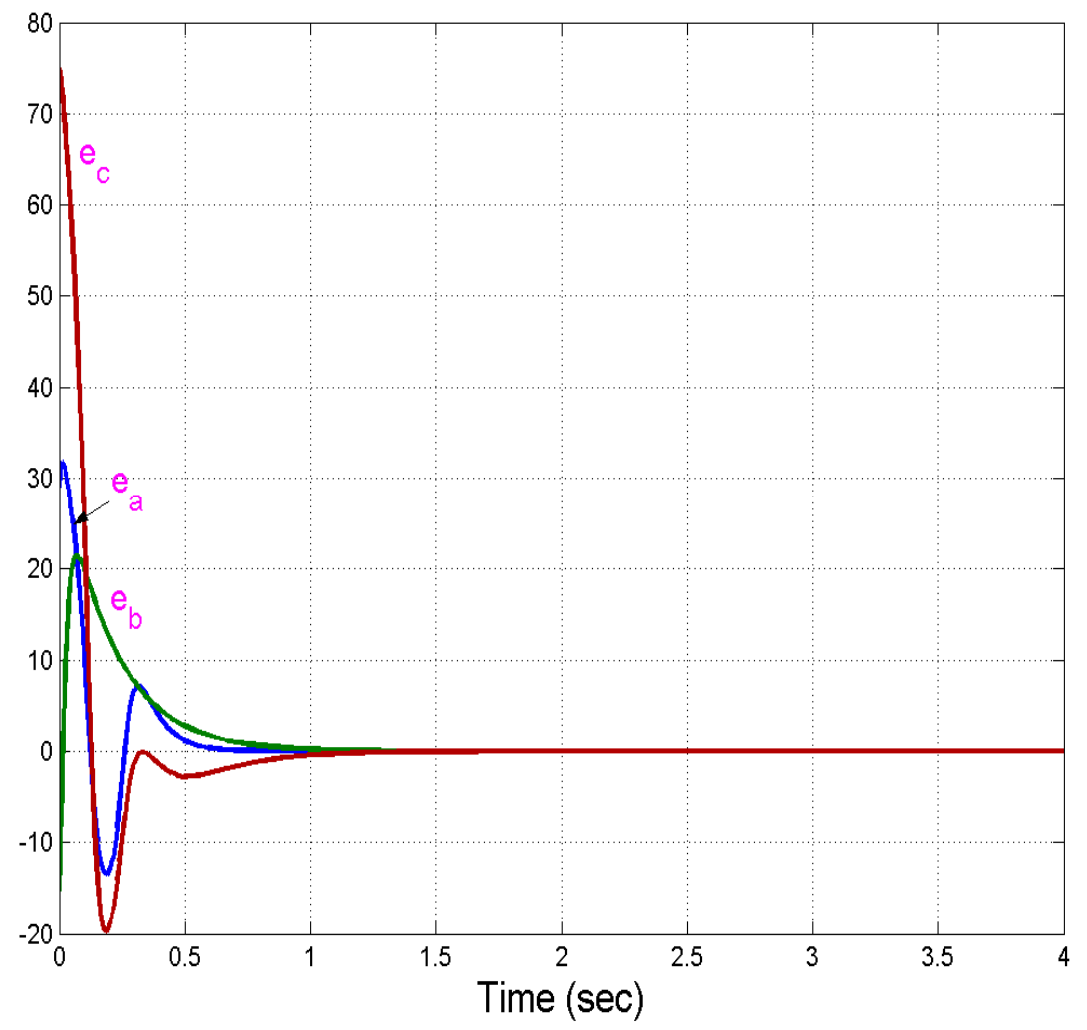

Figure 4. Time-History of the Parameter Estimates $e_{a}, e_{b}, e_{c}$

\section{Adaptive Synchronization of Identical Qi-Chen Chaotic SYSTEMS}

\subsection{Theoretical Results}

In this section, we discuss the adaptive synchronization of identical Qi-Chen chaotic systems (2005) with unknown parameters.

As the master system, we consider the Qi-Chen dynamics described by

$$
\begin{aligned}
& \dot{x}_{1}=a\left(x_{2}-x_{1}\right)+x_{2} x_{3} \\
& \dot{x}_{2}=c x_{1}-x_{2}-x_{1} x_{3} \\
& \dot{x}_{3}=x_{1} x_{2}-b x_{3}
\end{aligned}
$$

where $x_{i},(i=1,2,3)$ are the state variables and $a, b, c$ are unknown system parameters.

The system (13) is chaotic when the parameter values are taken as

$$
a=35, b=8 / 3 \text { and } c=80 \text {. }
$$


International Journal of Computer Science, Engineering and Applications (IJCSEA) Vol.2, No.1, February 2012

As the slave system, we consider the modified hyperchaotic Lü dynamics described by

$$
\begin{aligned}
& \dot{y}_{1}=a\left(y_{2}-y_{1}\right)+y_{2} y_{3}+u_{1} \\
& \dot{y}_{2}=c y_{1}-y_{2}-y_{1} y_{3}+u_{2} \\
& \dot{y}_{3}=y_{1} y_{2}-b y_{3}+u_{3}
\end{aligned}
$$

where $y_{i},(i=1,2,3)$ are the state variables and $u_{i},(i=1,2,3)$ are the nonlinear controllers to be designed.

The synchronization error is defined by

$$
e_{i}=y_{i}-x_{i}, \quad(i=1,2,3)
$$

Then the error dynamics is obtained as

$$
\begin{aligned}
& \dot{e}_{1}=a\left(e_{2}-e_{1}\right)+y_{2} y_{3}-x_{2} x_{3}+u_{1} \\
& \dot{e}_{2}=c e_{1}-e_{2}-y_{1} y_{3}+x_{1} x_{3}+u_{2} \\
& \dot{e}_{3}=-b e_{3}+y_{1} y_{2}-x_{1} x_{2}+u_{3}
\end{aligned}
$$

Let us now define the adaptive control functions $u_{1}(t), u_{2}(t), u_{3}(t)$ as

$$
\begin{aligned}
& u_{1}=-\hat{a}\left(e_{2}-e_{1}\right)-y_{2} y_{3}+x_{2} x_{3}-k_{1} e_{1} \\
& u_{2}=-\hat{c} e_{1}+e_{3}+y_{1} y_{3}-x_{1} x_{3}-k_{2} e_{2} \\
& u_{3}=\hat{b} e_{3}-y_{1} y_{2}+x_{1} x_{2}-k_{3} e_{3}
\end{aligned}
$$

where $\hat{a}, \hat{b}$ and $\hat{c}$ are estimates of the parameters $a, b$ and $c$, respectively, and $k_{i},(i=1,2,3)$ are positive constants.

Substituting the control law (17) into (16), we obtain the error dynamics as

$$
\begin{aligned}
& \dot{e}_{1}=(a-\hat{a})\left(e_{2}-e_{1}\right)-k_{1} e_{1} \\
& \dot{e}_{2}=(c-\hat{c}) e_{1}-k_{2} e_{2} \\
& \dot{e}_{3}=-(b-\hat{b}) e_{3}-k_{3} e_{3}
\end{aligned}
$$

Let us now define the parameter errors as

$$
\begin{aligned}
e_{a} & =a-\hat{a} \\
e_{b} & =b-\hat{b} \\
e_{c} & =c-\hat{c}
\end{aligned}
$$


Substituting (19) into (18), the error dynamics simplifies to

$$
\begin{aligned}
& \dot{e}_{1}=e_{a}\left(e_{2}-e_{1}\right)-k_{1} e_{1} \\
& \dot{e}_{2}=e_{c} e_{1}-k_{2} e_{2} \\
& \dot{e}_{3}=-e_{b} e_{3}-k_{3} e_{3}
\end{aligned}
$$

For the derivation of the update law for adjusting the estimates of the parameters, the Lyapunov approach is used.

Consider the quadratic Lyapunov function

$$
V\left(e_{1}, e_{2}, e_{3}, e_{a}, e_{b}, e_{c}\right)=\frac{1}{2}\left(e_{1}^{2}+e_{2}^{2}+e_{3}^{2}+e_{a}^{2}+e_{b}^{2}+e_{c}^{2}\right)
$$

which is a positive definite function on $R^{6}$.

Note also that

$$
\begin{aligned}
& \dot{e}_{a}=-\dot{\hat{a}} \\
& \dot{e}_{b}=-\dot{\hat{b}} \\
& \dot{e}_{c}=-\dot{\hat{c}}
\end{aligned}
$$

Differentiating $V$ along the trajectories of (20) and using (22), we obtain

$$
\dot{V}=-k_{1} e_{1}^{2}-k_{2} e_{2}^{2}-k_{3} e_{3}^{2}+e_{a}\left[e_{1}\left(e_{2}-e_{1}\right)-\dot{\hat{a}}\right]+e_{b}\left[-e_{3}^{2}-\dot{\hat{b}}\right]+e_{c}\left[e_{1} e_{2}-\dot{\hat{c}}\right]
$$

In view of Eq. (23), the estimated parameters are updated by the following law:

$$
\begin{aligned}
& \dot{\hat{a}}=e_{1}\left(e_{2}-e_{1}\right)+k_{4} e_{a} \\
& \dot{\hat{b}}=-e_{3}^{2}+k_{5} e_{b} \\
& \dot{\hat{c}}=e_{1} e_{2}+k_{6} e_{c}
\end{aligned}
$$

where $k_{4}, k_{5}$ and $k_{6}$ are positive constants.

Substituting (24) into (23), we get

$$
\dot{V}=-k_{1} e_{1}^{2}-k_{2} e_{2}^{2}-k_{3} e_{3}^{2}-k_{4} e_{a}^{2}-k_{5} e_{b}^{2}-k_{6} e_{c}^{2}
$$

From (25), we find that $\dot{V}$ is a negative definite function on $R^{6}$.

Thus, by Lyapunov stability theory [30], it is immediate that the synchronization error and the parameter error decay to zero exponentially with time for all initial conditions.

Hence, we have proved the following result. 
Theorem 2. The identical Qi-Chen systems (13) and (14) with unknown parameters are globally and exponentially synchronized for all initial conditions by the adaptive control law (17), where the update law for parameters is given by $(24)$ and $k_{i},(i=1, \ldots, 6)$ are positive constants.

\subsection{Numerical Results}

For the numerical simulations, the fourth order Runge-Kutta method is used to solve the two systems of differential equations (13) and (14) with the adaptive control law (17) and the parameter update law (24).

We take the parameter values as in the chaotic case, viz.

$$
a=35, \quad b=8 / 3, \quad c=80
$$

We take the positive constants $k_{i},(i=1, \ldots, 8)$ as

$$
k_{i}=5 \text { for } i=1,2, \ldots, 6 \text {. }
$$

Suppose that the initial values of the estimated parameters are

$$
\hat{a}(0)=12, \hat{b}(0)=9, \quad \hat{c}(0)=26
$$

We take the initial values of the master system (13) as

$$
x_{1}(0)=7, \quad x_{2}(0)=-5, \quad x_{3}(0)=16
$$

We take the initial values of the slave system (14) as

$$
y_{1}(0)=33, \quad y_{2}(0)=18, \quad y_{3}(0)=-7
$$

Figure 5 shows the adaptive chaos synchronization of the identical Qi-Chen systems.

Figure 6 shows the time-history of the synchronization error $e_{1}, e_{2}, e_{3}$.

Figure 7 shows the time-history of the parameter estimates $\hat{a}(t), \hat{b}(t), \hat{c}(t)$. From this figure, it is clear that the parameter estimates converge to the original values $a=35, b=8 / 3, c=80$, respectively.

Figure 8 shows the time-history of the parameter estimation errors $e_{a}, e_{b}, e_{c}$. 

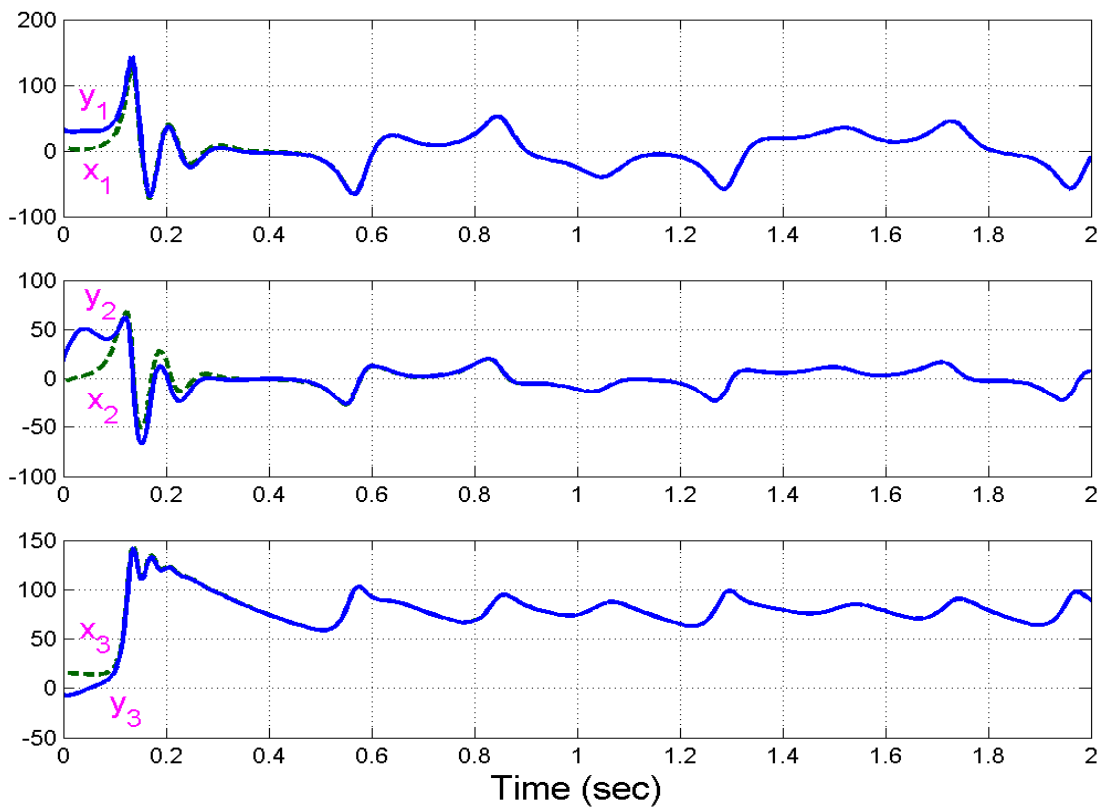

Figure 5. Adaptive Synchronization of the Qi-Chen Systems

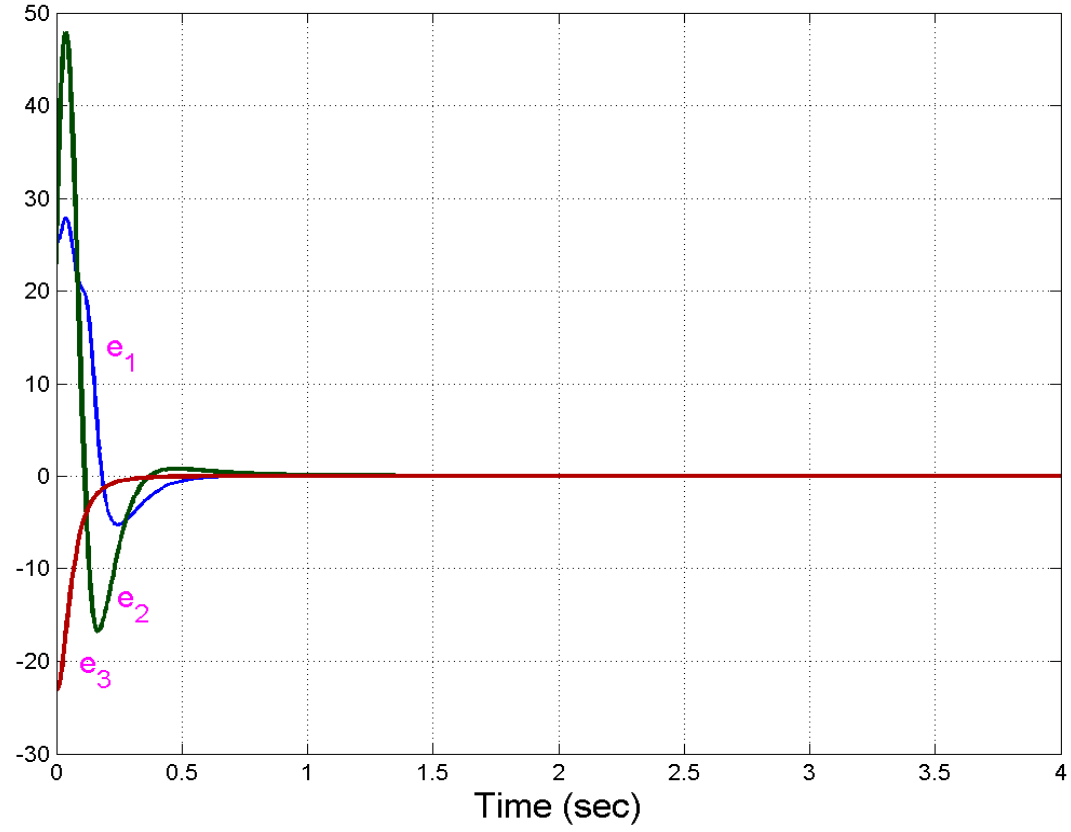

Figure 6. Time-History of the Synchronization Error $e_{1}, e_{2}, e_{3}$ 
International Journal of Computer Science, Engineering and Applications (IJCSEA) Vol.2, No.1, February 2012

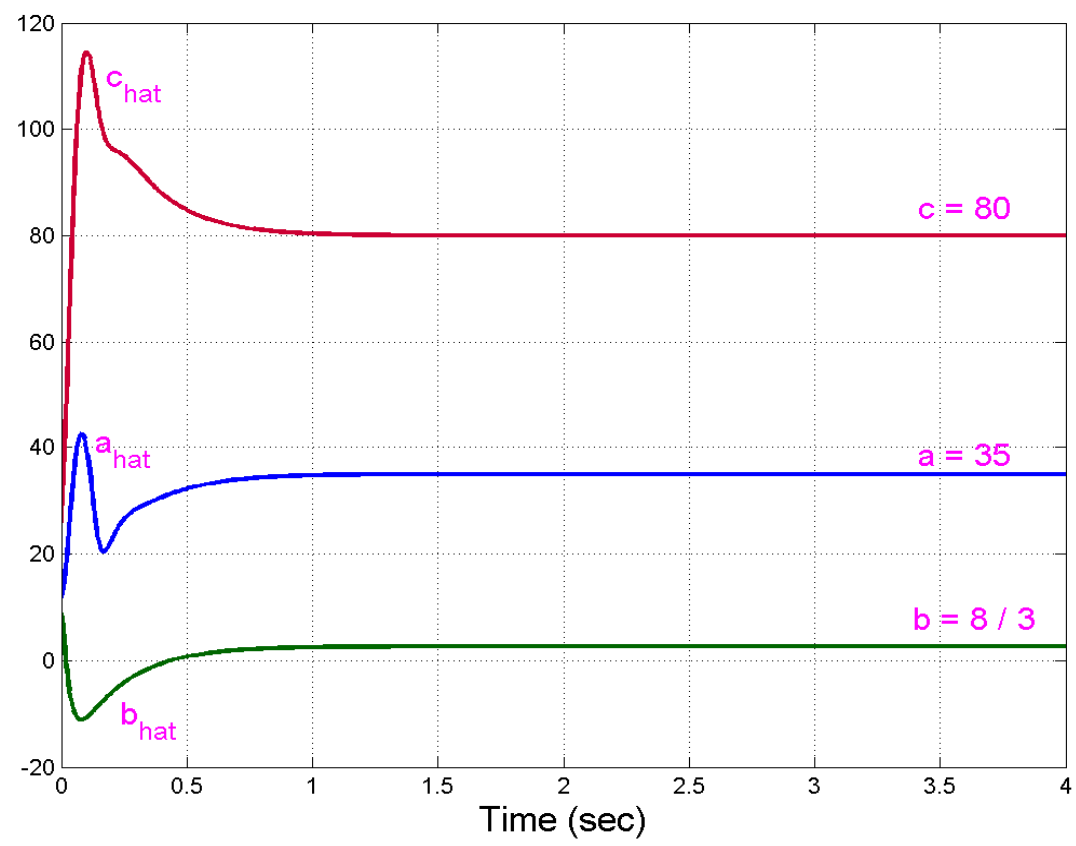

Figure 7. Time-History of the Parameter Estimates $\hat{a}(t), \hat{b}(t), \hat{c}(t)$

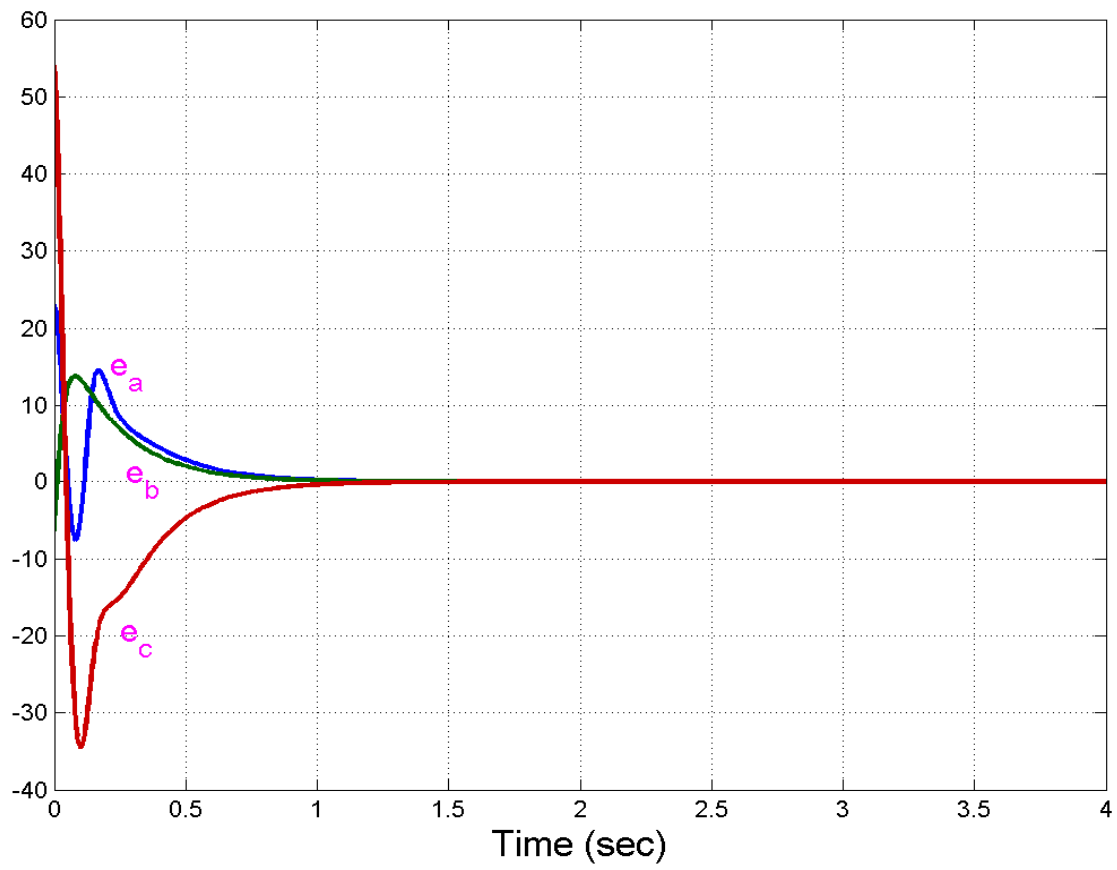

Figure 8. Time-History of the Parameter Estimation Error $e_{a}, e_{b}, e_{c}$ 


\section{Conclusions}

In this paper, we applied adaptive control theory for the stabilization and synchronization of the Qi-Chen system (2005) with unknown system parameters. First, we designed adaptive control laws to stabilize the Qi-Chen system to its unstable equilibrium point at the origin based on the adaptive control theory and Lyapunov stability theory. Then we derived adaptive synchronization scheme and update law for the estimation of system parameters for the identical Qi-Chen systems with unknown parameters. Our synchronization schemes were established using Lyapunov stability theory. Since the Lyapunov exponents are not required for these calculations, the proposed adaptive control method is very effective and convenient to achieve chaos control and synchronization of the Qi-Chen chaotic system. Numerical simulations are shown to demonstrate the effectiveness of the proposed adaptive stabilization and synchronization schemes.

\section{REFERENCES}

[1] Lorenz, E.N. (1963) "Deterministic nonperiodic flow," J. Atmos. Phys. Vol. 20, pp 131-141.

[2] Rössler, O.E. (1976) “An equation for continuous chaos,” Physics Letters A, Vol. 57, pp 397-398.

[3] Wang, X., Tian, L. \& Yu, L. (2006) "Adaptive control and slow manifold analysis of a new chaotic system,” Internat. J. Nonlinear Science, Vol. 21, pp 43-49.

[4] Sun, M., Tian, L., Jiang, S. \& Xun, J. (2007) "Feedback control and adaptive control of the energy resource chaotic system," Chaos, Solitons \& Fractals, Vol. 32, pp 168-180.

[5] Pecora, L.M. \& Carroll, T.L. (1990) "Synchronization in chaotic systems", Phys. Rev. Lett., Vol. 64, pp 821-824.

[6] Lakshmanan, M. \& Murali, K. (1996) Nonlinear Oscillators: Controlling and Synchronization, World Scientific, Singapore.

[7] Han, S.K., Kerrer, C. \& Kuramoto, Y. (1995) "Dephasing and bursting in coupled neural oscillators", Phys. Rev. Lett., Vol. 75, pp 3190-3193.

[8] Blasius, B., Huppert, A. \& Stone, L. (1999) "Complex dynamics and phase synchronization in spatially extended ecological system”, Nature, Vol. 399, pp 354-359.

[9] Feki, M. (2003) "An adaptive chaos synchronization scheme applied to secure communication", Chaos, Solitons and Fractals, Vol. 18, pp 141-148.

[10] Murali, K. \& Lakshmanan, M. (1998) "Secure communication using a compound signal from generalized synchronizable chaotic systems", Phys. Rev. Lett. A, Vol. 241, pp 303-310.

[11] Yang, T. \& Chua, L.O. (1999) "Control of chaos using sampled-data feedback control”, Internat. J. Bifurcat. Chaos, Vol. 9, pp 215-219.

[12] Ott, E., Grebogi, C. \& Yorke, J.A. (1990) “Controlling chaos”, Phys. Rev. Lett., Vol. 64, pp 11961199.

[13] Park, J.H. \& Kwon, O.M. (2003) "A novel criterion for delayed feedback control of time-delay chaotic systems", Chaos, Solitons and Fractals, Vol. 17, pp 709-716.

[14] Yu, Y.G. \& Zhang, S.C. (2006) "Adaptive backstepping synchronization of uncertain chaotic systems", Chaos, Solitons and Fractals, Vol. 27, pp 1369-1375.

[15] Ho, M.C. \& Hung, Y.C. (2002) "Synchronization of two different chaotic systems by using generalized active control”, Physics Letters A, Vol. 301, pp 424-428.

[16] Huang, L., Feng, R. \& Wang, M. (2004) "Synchronization of chaotic systems via nonlinear control", Physics Letters A, Vol. 320, pp 271-275.

[17] Tang, R.A., Liu, Y.L. \& Xue, J.K. (2009) "An extended active control for chaos synchronization," Physics Letters A, Vol. 373, No. 6, pp 1449-1454.

[18] Sundarapandian, V. (2011) "Output regulation of the Sprott-G chaotic system by state feedback control”, International Journal of Instrumentation and Control Systems, Vol. 1, No. 1, pp 20-30.

[19] Sundarapandian, V. (2011) "Global chaos synchronization of Lorenz and Pehlivan chaotic systems by nonlinear control", International Journal of Advances in Science and Technology, Vol. 2, No. 3, pp 19-28.

[20] Lei, Y., Xu, W. \& Zheng, H. (2005) "Synchronization of two chaotic nonlinear gyros using active control," Physics Letters A, Vol. 343, No. 1, pp 153-158. 
[21] Liao, T.L. \& Tsai, S.H. (2000) "Adaptive synchronization of chaotic systems and its applications to secure communications", Chaos, Solitons and Fractals, Vol. 11, pp 1387-1396.

[22] Park, J.H., Lee, S.M. \& Kwon, O.M. (2007) "Adaptive synchronization of Genesio-Tesi chaotic system via a novel feedback control," Physics Letters A, Vol. 371, pp 263-270.

[23] Sundarapandian, V. (2011) "Adaptive control and synchronization of hyperchaotic Chen system", International Journal of Information Technology Convergence and Services, Vol. 1, No. 3, pp 22-33.

[24] Sundarapandian, V. (2011) "Adaptive synchronization of hyperchaotic Lorenz and hyperchaotic Lü systems", International Journal of Instrumentation and Control Systems, Vol. 1, No. 1, pp 1-18.

[25] Sundarapandian, V. (2011) "Adaptive control and synchronization of hyperchaotic Cai system", International Journal of Control Theory and Computer Modeling, Vol. 1, No. 1, pp 1-13.

[26] Konishi, K., Hirai, M. \& Kokame, H. (1998) "Sliding mode control for a class of chaotic systems", Physics Letters A, Vol. 245, pp 511-517.

[27] Sundarapandian, V. (2011) "Sliding mode controller design for synchronization of Shimizu-Morioka chaotic system”, International Journal of Information Sciences and Techniques, Vol. 1, No. 1, pp 2029.

[28] Sundarapandian, V. (2011) "Global chaos synchronization of four-wing chaotic systems by sliding mode control", International Journal of Control Theory and Computer Modeling, Vol., 1 No. 1, pp 15-31.

[29] Qi, G., Chen, G., Du, S., Chen, Z. and Yuan, Z. (2005) “Analysis of a new chaotic system," Physica A, Vol. 352, pp 295-308.

[30] Hahn, W. (1967) The Stability of Motion, Springer, New York.

\section{Author}

Dr. V. Sundarapandian obtained his Doctor of Science degree in Electrical and Systems Engineering from Washington University, St. Louis, USA in May 1996. He is a Professor at the R \& D Centre at Vel Tech Dr. RR \& Dr. SR Technical University, Chennai, Tamil $\mathrm{Nadu}$, India. He has published over 230 refereed international publications. He has published over 150 papers in National and International Conferences. He is the Editor-inChief of the AIRCC Journals - International Journal of Instrumentation and Control Systems, International Journal of Control Systems and Computer Modelling, and

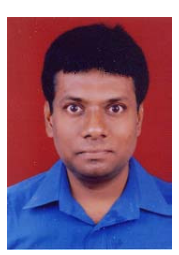
International Journal of Information Technology, Control and Automation. His research interests are Linear and Nonlinear Control Systems, Chaos Theory and Control, Soft Computing, Optimal Control, Operations Research, Mathematical Modelling and Scientific Computing. He has delivered several Key Note Lectures on Control Systems, Chaos Theory and Control, Scientific Computing using MATLAB/SCILAB, etc. 